\title{
Advancements in Stereotactic Epilepsy Surgery: Stereo-EEG, Laser Interstitial Thermotherapy, and Responsive Neurostimulation
}

\author{
Michael J. Lang, MD; Ameet Chitale, MD; Ashwini D. Sharan, MD; \\ Chengyuan Wu, MD
}

Department of Neurological Surgery, Thomas Jefferson University, Philadelphia, PA

\begin{abstract}
Stereotactic interventions form an increasingly significant portion of the minimally invasive approaches for surgical management of epilepsy. ${ }^{1,2}$ This manuscript will review the application of three recent stereotactic techniques in the modern epilepsy surgery armamentarium, namely stereotactic electroencephalography (SEEG), responsive neural stimulation (RNS) and laser interstitial thermal therapy (LITT). While these interventions are a contemporary advancement, they are intellectually indebted to some of the most major developments and pioneers in the history of neurosurgery. Sir Victor Horsley, the father of modern neurosurgery, and Robert Clarke developed the first stereotactic frame in 1908, but use of the stereotactic coordinate space did not find wide use until it could be paired with intracranial imaging. Acquisition of pneumoencephalograms and/ or arterial angiography (developed by Dandy and Moniz, respectively) with a stereotactic reference frame enabled Spiegel and Wycis to precisely localize brain structures. ${ }^{3}$ The ability to attain sub-millimeter accuracy followed the advent of computed tomography (CT) and magnetic resonance imaging (MRI). These advancements were applied to epilepsy first by Bancaud and Talairach with their development of SEEG. ${ }^{4}$ While LITT and RNS represent more recent advancements, they are indebted to the work of Lars Leksell and Alim Benabid for their pioneering work in stereotactic ablative therapy and deep brain stimulation (DBS), respectively.
\end{abstract}

\section{STEREOTACTIC EEG}

As with all surgical interventions for epilepsy, the decision to pursue SEEG recording must serve to test an anatomo-electro-clinical (AEC) hypothesis. ${ }^{5}$ At Thomas Jefferson, a multi-disciplinary surgical epilepsy conference, including clinicians from the departments of Neurology, Neurosurgery, Neuropsychology, and Neuroradiology, evaluates all potential surgical candidates. Invasive intracranial monitoring is indicated in cases in which phase I monitoring with surface EEG fails to lateralize and/or localize a presumed focal epileptic onset zone, particularly in the context of discordant data derived from EEG, MRI, neuropsychologic testing, and functional imaging such as positron emission tomography (PET). It cannot be over-emphasized that decision-making with regard to intracranial implant strategy must serve to test the AEC hypothesis, and so-called "fishing expeditions" should be avoided at all costs.

Historically, intracranial monitoring has developed along two major pathways, strip/ grid electrodes placed over the cortical surface or depth electrodes placed into brain tissue itself. Following its introduction by Talairach and Bancaud in the 1950's, SEEG has seen widespread use in European epilepsy centers. However, initial safety concerns and the complexity associated with surgical planning prior to modern multimodal imaging limited its use in North America, resulting in a general preference for subdural strip and grid electrodes. ${ }^{6,7}$ With substantial evidence regarding safety and clinical utility, there has been a significant resurgence of interest in SEEG in North America in the last decade. Stereotactic EEG offers several distinct advantages relative to subdural strip/grid recordings. First, SEEG offers the ability to record from deep-seated structures that are poorly interrogated by scalp or strip/grid electrodes, such as subdivisions of the mesial temporal structures, cingulate gyrus, insula/operculum, and the depths of sulci. Second, individually tailored implants may be designed to interrogate network structures up- and down-stream from the presumed symptomatogenic zone. ${ }^{8,9}$ Furthermore, our group has found distinct advantages with SEEG in terms of safety and patient comfort for large bihemispheric implantations and in patients with prior history of craniotomy. Finally, SEEG allows for the generation of a four dimensional picture of epileptogenic zone and spread patterns, which is not reliably achieved with cortical surface recording techniques. As such, our center is actively pursuing SEEG-based white-matter recording guided by functional imaging to further define cerebral networks and spread patterns in epilepsy.

At Jefferson, SEEG implantation planning is performed with the use of co-registered multimodal imaging. Imaging is obtained at least two weeks prior to surgery in order to facilitate the complex nature of surgical planning. High-resolution gadolinium-enhanced T1-weighted images with sub-millimeter isometric voxels (to minimize spatial warping during multiplanar reconstruction) serve as the base imaging set. Additional MR sequences are co-registered as required, such as tractography and fMRI. In order to minimize the risk of vascular injury, catheter angiography is acquired, and a threedimensional cone-beam angiographic CT is reconstructed using Siemens DynaCT (Siemens, Munich, Germany) technology. This imaging technique provides better definition of small vessels in the late arterial and early venous phases that are poorly identified with traditional CT angiography. Furthermore, CT imaging of the calvarium improves the ability to avoid cranial defects from prior surgery and regions of skull too thin to effectively anchor the electrode bolt.

The implant plan is individualized on the basis of the AEC hypothesis, but typically comprises 8-16 electrodes. In general, trajectory planning for SEEG utilizes typical rules for stereotaxy, including entry points along gyral crowns, avoidance of vessels, sulci and ventricle ependymal surfaces. Full discussion of the nuances of trajectory planning for interrogation of all relevant supratentorial structures with potential epileptogenic potential 


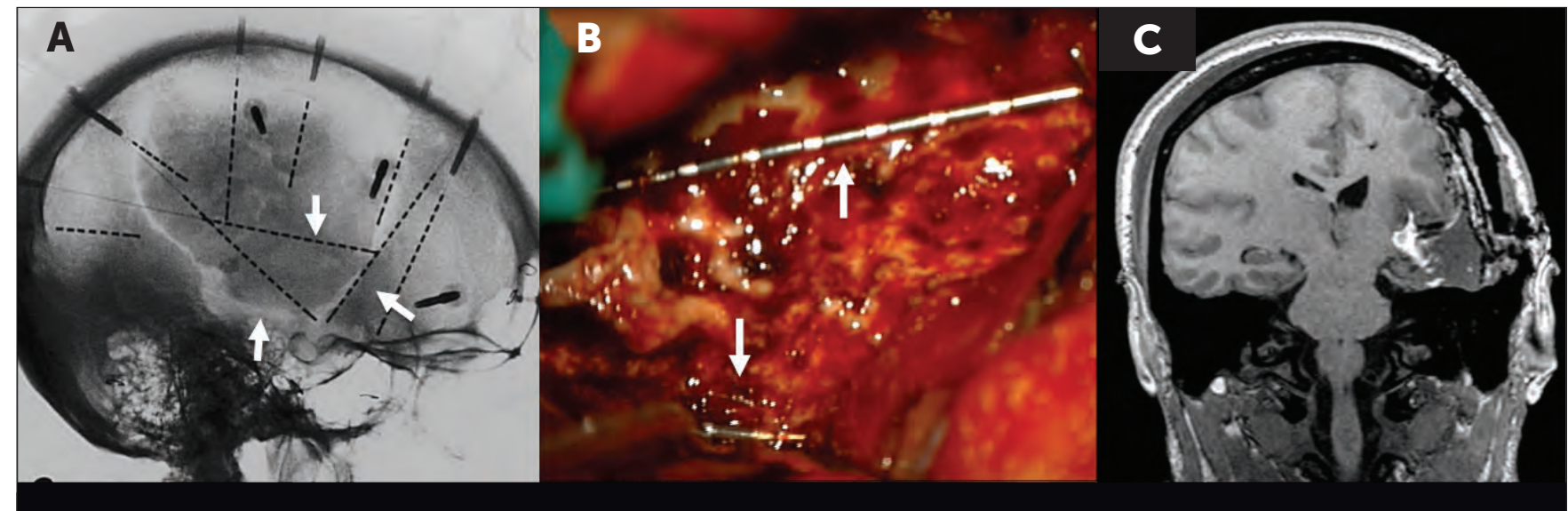

Figure 4

A 37 year-old female with a history of complex partial seizures secondary generalization had undergone prior temporal lobectomy for presumed mesial temporal onset based on scalp EEG, with no change in seizure character or frequency post-operatively. She subsequently underwent SEEG implantation (A), including a novel technique for insular lobe recordings along the triangular borders of the insular cortex (arrows). Ictal recordings demonstrated broad insular onset, for which the patient underwent planned subtotal insular resection (B). Given the oblique trajectory and entry outside of the planned craniotomy, the insular triangulation electrodes were left in place during surgery in order to aid identification of the insular borders. Post-operative MR imaging demonstrated the insular resection (C).

is beyond the scope of this article, but several features unique to SEEG planning deserve mention. Stereo-EEG enables recording of the entire cortical grey matter from gyrus to sulcal depth, so it may be advantageous in certain situations for entry to be slightly off the gyral crown. Ideally, trajectories should be planned orthogonal to the skull surface in order to avoid drill skiving, but highly oblique trajectories may prove useful, especially when interrogation of discrete surface and deep structures can be achieved with a single electrode. SEEG also requires significantly more trajectories per patient than typical applications for frame-based stereotactic surgery (such as DBS or needle biopsy). As such, planning requires careful attention in avoiding electrode collision. Furthermore, thought must be given to approximate location of fixation pins on the stereotactic frame during planning, so that they do not interfere with planned trajectories after frame placement. For this reason, we use the CRW frame (Integra, Plainsboro, $\mathrm{NJ}$ ) during SEEG procedures because of the increased freedom in pin placement compared to other stereotactic frames. Following initial completion, trajectories are evaluated for safety and adequacy of hypothesis testing within the AEC framework in a meeting of all members of the Epilepsy and Neuromodulation Neurosurgery team.
On the day of surgery, the CRW stereotactic frame is applied under local anesthesia to facilitate ideal pin positioning in an awake, cooperative patient. High-resolution three-dimensional noncontrast CT imaging is acquired with the localizer frame to define stereotactic space, and pre-operative images (with planned trajectories) are co-registered to the sterotactic scan. Anesthesia is induced after awake fiber-optic intubation. During this time, the NeuroMate stereotactic robotic platform (Renishaw, Gloucester, UK) is set up and calibration tests are performed to ensure accuracy of the system prior to initiation of the procedure. Once the anesthetized patient is fixed to the robot frame attachment, accuracy is again confirmed using test trajectories (with $2 \mathrm{~mm}$ error being considered maximum tolerance, though in practice submillimeter accuracy is maintained). The robot is used in place of a manual stereotactic arc to orient instruments along the planned trajectory. Anchor bolts are placed percutaneously, using a $2.0 \mathrm{~mm}$ non-skiving drill bit (SurgiBit, Sydney, Australia) to slightly under-drill the hole for electrode anchor bolts with $2.1 \mathrm{~mm}$ outer diameter (PMT Corp, Chanhassen, MN), which is placed after cautery of the dura/pia with an insulated ablation probe (PMT Corp, Chanhassen, $\mathrm{MN})$. Following placement of all anchor bolts, the $\mathrm{O}$-Arm intra-operative $\mathrm{CT}$ scanner (Medtronic Inc., Minneapolis, $\mathrm{MN}$ ) is draped and brought into the field. The O-Arm is used to obtain plain x-ray images of a guiding stylette placed to the planned target, and final position of the electrode (PMT Corp, Chanhassen, MN), is then confirmed relative to images with the stylette. Active recording length of the electrode is determined by the number of contacts (available in even increments from 8 to 16 contacts), based on pre-operatively planned targets. After all electrodes are placed, the O-Arm is used to obtain a three-dimensional CT scan, which is co-registered to pre-operative imaging to confirm accuracy of electrode placement.

As worldwide experience with SEEG has increased, the relative advantages and disadvantages of the technique have emerged. Concerns with regard to the safety of SEEG implantation, particularly within the North American literature, ${ }^{10}$ have been addressed. A recent metaanalysis of the reported intracranial monitoring literature by Mullin et al. showed that SEEG has a better safety profile compared to subdural grid or strip recording, with an overall complication rate of $1.3 \%$ compared to $4.0 \%$ with subdural recordings. ${ }^{11}$ Subdural electrodes were shown to have statistically significant increased risk of both hemorrhagic and infectious complications. That said, both techniques have 


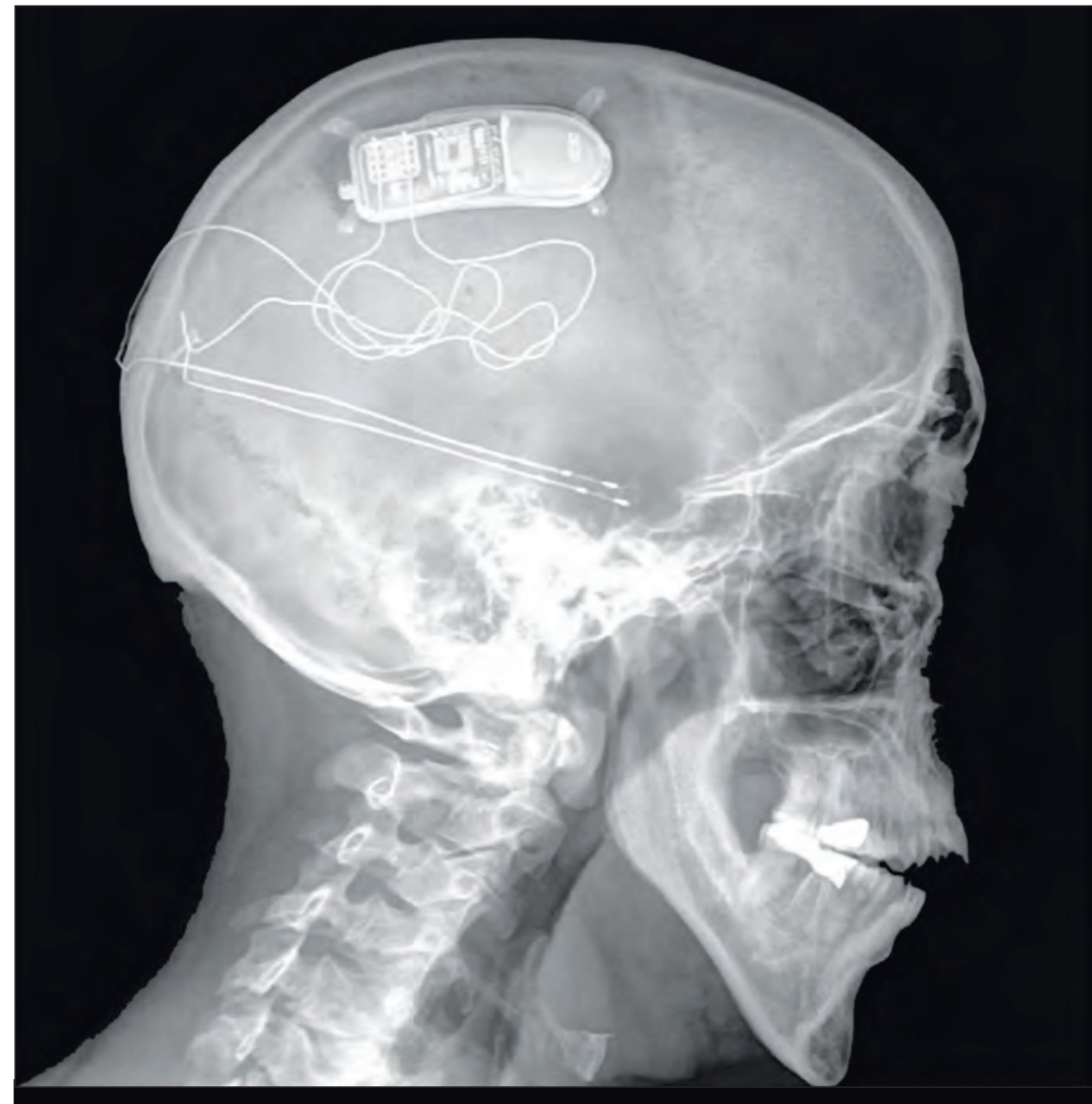

\section{Figure 2}

Ictal onset in a 46 year-old male with complex partial seizures was unable to be localized or lateralized on the basis of scalp EEG monitoring. He subsequently underwent SEEG implantation, including bitemporal electrode implantation, and was found to have seizures originating independently in the mesial temporal lobes bilaterally. Both hippocampi were cannulated along their long axes and 4 contact depth electrodes were connected to the NeuroPace device, which is anchored in the skull following a craniectomy.

demonstrated similar very low rates of mortality and permanent morbidity $(0.5$ $0.6 \%$ ). While SEEG has shown benefit in recording deep structures, many authors have suggested that grid implantation continues to show benefit in mapping of functional cortex (particularly wernicke's area), though techniques to facilitate brain mapping with SEEG continue to be developed. ${ }^{12,13}$

In our experience to date at Jefferson, 27 patients have undergone implantation of a total of 342 electodes (mean 12.7, range 5-17). No patient experienced permanent morbidity or mortality related to the SEEG hemorrhage in the anterior temporal white matter. All patients with surgically resectable focal epilepsy have undergone subsequent resective or ablative surgery. Five patients in this series have undergone RNS as a result of SEEG implantation, due to identification of bitemporal or eloquent cortex EZ.

\section{LASER INTERSTITIAL THERMOTHERAPY}

Laser ablation has arisen in response to two older minimally invasive ablative techniques: radiofrequency (RF) thermocoagulation and stereotactic radiosurgery (SRS). Thermal ablation for focal epilepsy was initially described using radiofrequency (RF) thermocoagulation probes. However, while the thermocouple feature of RF probes allows for thermal monitoring at the electrode tip, this technique does not allow for monitoring of the ablation zone as it expands, particularly in relation to the volume of interest and adjacent eloquent structures. Radiosurgical treatment of epileptogenic foci has shown efficacy, but must contend with radiobiologic complications such as radionecrosis, as well as the inherently delayed effect of treatment. As such, LITT has emerged as a therapeutic modality by which heat deposition into tissue can be monitored in real time in the MRI environment. Laser energy is absorbed in a variable manner depending on wavelength, tissue characteristics, and proximity to diffusive heat sinks such as CSF spaces and blood vessels. Alterations in proton resonant frequency can be used to extract temperature dependent component of tissue chemical shift in gradient recalled echo (GRE) sequences, resulting in real-time measurement of tissue temperatures within $1^{\circ} \mathrm{C} .{ }^{14}$ As a result, MRI can be used to identify the precise location of a stereotactically placed laser probe, and monitor tissue heating during laser energy deposition. Lesion growth is estimated as heating continues, with temperatures above $60^{\circ} \mathrm{C}$ resulting in instantaneous cell death and time/temperature dependent cell death for temperatures between $45-60^{\circ} \mathrm{C}$. Since the introduction of LITT, the technique has been increasingly used for both oncology and epilepsy neurosurgical indications. ${ }^{15,16}$ 

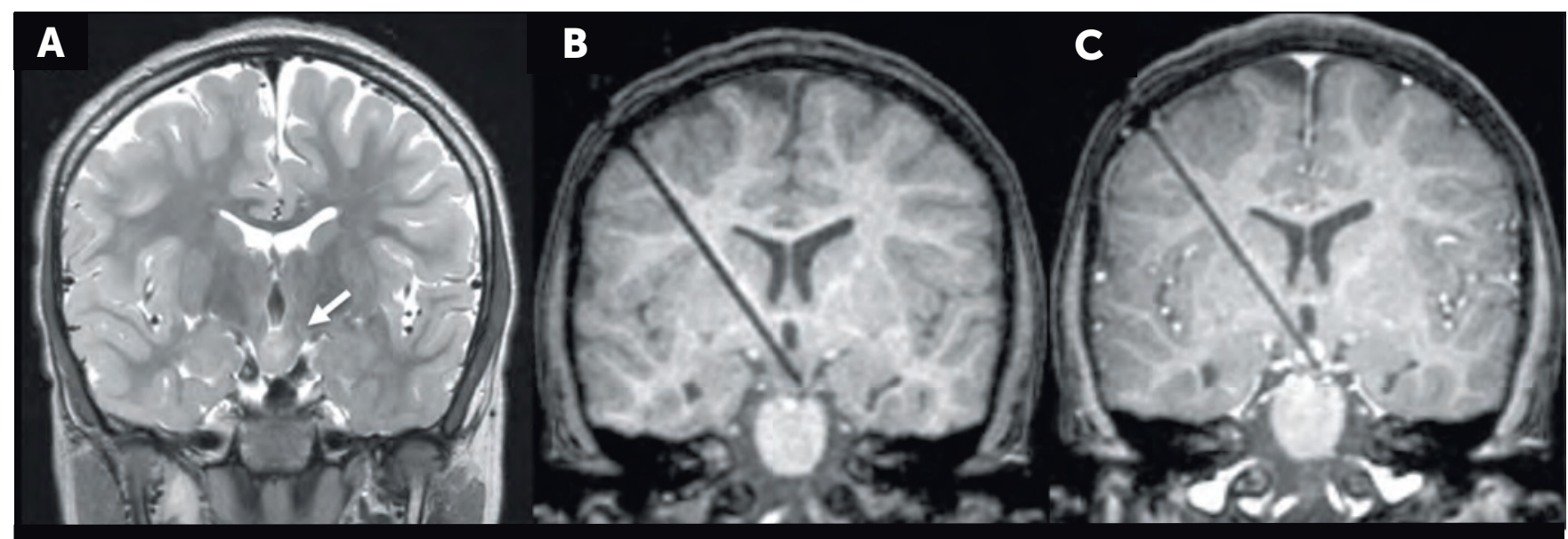

Figure 4

A 7-year old patient with gelastic seizures and precocious puberty was found to have a large hypothalamic hamartoma A. T2-weighted image). Given the significant morbidity associated with open surgical resection or detachment of these lesions, the patient was recommended for LITT. Intra-procedural MRI shows the accuracy of the stereotactic laser probe placement of the laser probe $\mathbf{B}$. T-1-weighted image without contrast). Real-time thermography guided ablation of the lesion, while preventing unintended heat deposition into the nearby hypothalamus. Post-contrast imaging confirmed the ablation of the hamartoma, including its attachment on the left hypothalamus.

Surgical planning for laser ablation varies with target, but in the Jefferson experience, targeting the amygdalohippocampal complex for mesial temporal sclerosis (MTS) is the most common indication. Long-axis cannulation of the mesial structures is planned from a posterior entry point, which typically is found within a $1 \mathrm{~cm}$ radius of a point $5 \mathrm{~cm}$ superior and lateral to the inion. The standard trajectory takes an extraventricular course in the white matter lying between the collateral sulcus and the floor of the lateral ventricle. Cerebrospinal fluid within the basal cisterns and the ventricle also acts as a heat sink to constrain thermal injury within the mesial structures. A relative trade-off exists between the volume of amygdala ablated and the posterior extent of the hippocampal body/fornix ablation, which is adjusted individually based on anatomic and semiological findings. An anatomic study by Wu et al. elucidates several of the nuances of LITT trajectory planning for MTLE in further detail. ${ }^{17}$ Intra-operatively, a manual arc-ring system is used with the CRW frame to guide placement of the anchor bolt, instead of the robotic system, given the time involved setting up the robot is not justified for a single trajectory. After drilling placement of a bone anchor bolt, a laser probe is advanced to target. In MTS cases, we have found that advancing a rigid stylette to target prior to placement of the laser probe results in less deviation by creating a tract in the firm, gliotic hippocampus. The patient is then transported to MRI (though intra-operative MRI is very well suited to this technique, if available), and the thermal ablation performed during real time thermography, mapped onto anatomic T1-weighted images.

In the epilepsy population, LITT has proved most beneficial in patients with MTS, hypothalamic hamartoma $(\mathrm{HH})$, and deep nodular heterotopias. In one series of 13 adult patients with mesial temporal lobe epilepsy (MTLE) with or without MTS, LITT resulted in approximately $60 \%$ destruction of the amygdalohippocampal volume and $54 \%$ seizure freedom at a mean follow-up of 14 months. ${ }^{15}$ Our own institutional experience has demonstrated similar results, with $53 \%$ seizure freedom at 6 months, $36.4 \%$ at 1 year, and $60 \%$ at 2-year follow-up (loss to follow-up in a small patient sample resulting in the variable results within the study period). ${ }^{18}$ Results in pediatric patients harboring $\mathrm{HH}$ have shown significantly lower postoperative complications compared to open surgical resection. In the Jefferson practice, laser amygdalohippocampectomy is offered instead of open selective amygdalohippocampectomy
(SAH) for patients with MTS and select MTLE patients. While early experience has shown a decreased rate of seizure freedom compared to anterior temporal lobectomy (ATL), the minimally invasive technique, sparing of neocortical structures, and relatively high success rate have made it an appealing option for these patients. Patients are counseled that salvage ATL is still possible in the event of failed laser ablation, with complete seizure freedom in $75 \%$ of patients requiring salvage $A T L$ in our experience.

\section{RESPONSIVE NEUROSTIMULATION}

Responsive neurostimulation is a variation on traditional neurostimulation paradigms such as spinal cord stimulation (SCS), deep brain stimulation (DBS), and vagal nerve stimulation (VNS). However, unlike these other forms of neurostimulation, which deliver current continuously, the NeuroPace device (NeuroPace, Mountain View, CA) delivers current only in response to a detected seizure, effectively prolonging battery life and minimizing side effects. In order to do so, the device captures electrocorticography (ECoG) from up to 8 channels via strip, depth, or combined strip and depth electrode implants attached to the device. The patient is taught to download ECoG 
recordings following a seizure so that the treating Neurologist can "teach" the device to recognize ictal onset and deliver a current impulse in order to terminate the seizure. This effectively uses the patient's unique seizure as an electrical biomarker to govern neurostimulation treatment. Following the results of the Pivotal trial, the device received FDA approval in 2013 for treatment of partial epilepsy.

As mentioned above, the device accommodates two 4-contact implanted electrodes, either strip, depth, or a combination. This modular configuration allows the implant to be tailored to a given patient. One common implant strategy includes bi-hippocampal depth electrodes for independent, bitemporal epilepsy, with long-axis cannulation as described above for laser ablation. For patients with seizures arising from a solitary temporal lobe after contralateral ATL, a hippocampal depth electrode is paired with a posterior basal sub-temporal strip electrode in an attempt to electrically trap outflow from the temporal lobe. In the case of seizures arising from the paracentral lobule, a pair of subdural strips over the medial and lateral sensorimotor cortices is often employed. A craniotomy is performed so that the implantable pulse generator can be anchored to the skull while sitting flush with the outer table. The cranial incision is planned such that only a small part of the incision requires re-opening when the battery has to be changed, and the electrode wires are tunneled so as to avoid crossing this segment of the incision, reducing risk of damage during re-operation.

The results of the Pivotal Trial showed the effect of RNS in a cohort of 191 patients with drug-resistant epilepsy. ${ }^{19}$ During the 12-week blinded treatment period, patients were randomized to responsive stimulation or seizure detection only. During this phase of the study, stimulated patients reported a decrease in seizure frequency of $37 \%$ in the stimulation group compared to $17 \%$ in the detection-only group, however both groups reported similar rates of $>50 \%$ reduction $(29 \%$ vs $27 \%)$. Interestingly, in the following openlabel phase of the trial, response rates increased gradually over the course of two years, with $44 \%$ and $53 \%$ seizure reduction at 1- and 2-year follow-up, respectively. ${ }^{20}$ Surgical complications included surgical site infections in $3 \%$ of patients, $6 \%$ rate of stimulation-induced paresthesias, and approximately $5 \%$ of patients reporting increase in both partial and generalized seizures. Overall, the benefit lies with its adaptability, but given the very low rate of seizure freedom with RNS, it should be considered alongside VNS and DBS as a palliative neurostimulation option for epilepsy. Continued development of the NeuroPace device, particularly an increase in the number of available channels, as well as increasing experience in patient selection and implant configuration may well improve outcomes with RNS over time.

\section{CONCLUSION}

Stereotactic surgical techniques make up an increasingly important component of the interventions for drug-resistant epilepsy. In particular, the complexity of trajectory planning greatly exceeds other stereotactic applications given the need to tailor depth electrode monitoring, ablative therapy, or neurostimulation therapy to the individual patient. Finally, every intervention should be carefully considered as part of coordinated, multi-specialty care, which develops anatomo-electrico-clinical hypotheses that are tested and treated in a patientspecific manner.

\section{REFERENCES}

1. Quigg M, Harden C. Minimally invasive techniques for epilepsy surgery: stereotactic radiosurgery and other technologies. Journal of Neurosurgery 2014;121 Suppl 2:232-40.

2. Chang EF, Englot DJ, Vadera S. Minimally invasive surgical approaches for temporal lobe epilepsy. Epilepsy \& Behavior 2015;47(C):24-33.

3. Rahman M, murad GJA, Mocco J. Early history of the stereotactic apparatus in neurosurgery. Neurosurg Focus 2009;27(3):1-5.

4. Bancaud J, Talairach J. La stéréo-électroencéphalographie dans l'épilepsie. Paris: Masson et Cie; 1965.

5. González-Martínez J, Mullin J, Bulacio J, et al. Stereoelectroencephalography in Children and Adolescents With Difficult-to-Localize Refractory Focal Epilepsy. Neurosurgery 2014;75(3):258-68.

6. Spencer SS, Spencer DD, Williamson PD, Mattson R. Combined depth and subdural electrode investigation in uncontrolled epilepsy. Neurology 1990;40(1):74-4.
7. Blatt DR, Roper SN, Friedman WA. Invasive monitoring of limbic epilepsy using stereotactic depth and subdural strip electrodes: surgical technique. Surgical Neurology 1997; 48:74-9.

8. Dylgjeri S, Taussig D, Chipaux M, et al. Insular and insulo-opercular epilepsy in childhood: an SEEG study. Seizure 2014;23(4):300-8.

9. González-Martínez J, Mullin J, Vadera S, et al. Stereotactic placement of depth electrodes in medically intractable epilepsy. Journal of Neurosurgery 2014;120(3):639-44.

10. Dorfer C, Stefanits H, Pataraia E, et al. Frameless Stereotactic Drilling for Placement of Depth Electrodes in Refractory Epilepsy. Neurosurgery 2014;10:582-91.

11. Mullin JP, Shriver M, Alomar S, et al. Is SEEG safe? A systematic review and meta-analysis of stereo-electroencephalography-related complications. Epilepsia 2016;57(3):386-401.

12. Mullin JP, Sexton D, Al-Omar S, Bingaman W, González-Martínez J. Outcomes of Subdural Grid Electrode Monitoring in the Stereoelectroencephalography Era. World Neurosurgery 2016;89(C):255-8.

13. Munyon C, Sweet J, Luders $H$, Lhatoo S, Miller J. The 3-Dimensional Grid: A Novel Approach to Stereoelectroencephalography. Neurosurgery 2015;

14. quesson B, de Zwart JA, Moonen CTW. Magnetic Resonance Temperature Imaging for Guidance of Thermotherapy. Journal of Magnetic Resonance Imaging 2000;12:525-33.

15. Willie JT, Laxpati NG, Drane DL, et al. Realtime magnetic resonance-guided stereotactic laser amygdalohippocampotomy for mesial temporal lobe epilepsy. Neurosurgery 2014;74(6):569-84-discussion584-5.

16. Brogan SE, Winter NB, Abiodun A. A cost utilization analysis of intrathecal therapy for refractory cancer pain: identifying factors associated with cost benefit. Pain Medicine 2013

17. Wu C, LaRiviere MJ, Laxpati N, Evans JJ, Gross RE, Sharan AD. Extraventricular LongAxis Cannulation of the Hippocampus. Neurosurgery 2014;10:325-33.

18. Kang JY, Wu C, Tracy J, et al. Laser interstitial thermal therapy for medically intractable mesial temporal lobe epilepsy. Epilepsia $2015 ;: n / a-n / a$

19. Morrell MJ, On behalf of the RNS System in Epilepsy Study Group. Responsive cortical stimulation for the treatment of medically intractable partial epilepsy. Neurology 2011;77(13):1295-304

20. Heck CN, King-Stephens D, Massey AD, et al. Two-year seizure reduction in adults with medically intractable partial onset epilepsy treated with responsive neurostimulation: Final results of the RNS System Pivotal trial. Epilepsia 2014;55(3):432-41. 